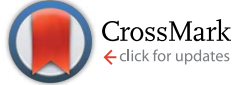

Cite this: RSC Adv., 2016, 6, 74506

Received 20th June 2016 Accepted 29th July 2016

DOI: $10.1039 / c 6 r a 15966 d$

www.rsc.org/advances

\section{Role of flexible bulky groups and weak interactions involving halogens in the vapoluminescence of a metal-free dye $\uparrow$}

\author{
Y. Akune, ${ }^{a}$ R. Hirosawa, ${ }^{a}$ H. Takahashi, ${ }^{b}$ M. Shiro ${ }^{c}$ and S. Matsumoto*a
}

A metal-free pyrazine dye exhibited vapoluminescence when exposed to benzene vapour. Single-crystal X-ray analysis revealed that its flexible bulky groups and weak interactions involving halogens play an essential role in the occurrence of the observed vapoluminescence.

Materials showing a change in their optical properties in response to external stimuli have attracted attention due to their potential application as sensor, switching, and memory devices. ${ }^{1}$ Among the various external stimuli, the change of colour upon exposure to solvent vapours, called vapochromism, can be used to detect volatile organic compounds (VOCs) in the environment and develop novel functional devices. Moreover, several VOC components of human breath are considered to be biomarkers for diseases such as cancer, ${ }^{2}$ and thus vapochromic materials might also enable easier disease diagnosis. Many of the reported vapochromic materials contain metal atom(s) and can be termed organometallic compounds, whereas few metalfree organic compounds have been reported. ${ }^{3}$ To improve the versatility of these materials, it is important to develop organic vapochromic compounds. Effective strategies for the molecular design of organic vapochromic materials were proposed in the scarce reports containing complete structural data for the vapour reaction. For example, alteration of intermolecular interactions, such as the $\pi-\pi$ interaction, ${ }^{4}$ and the interactions between donor and acceptor substituents by inclusion of solvent molecules, ${ }^{5}$ in addition to host-guest interactions, ${ }^{6}$ plays an essential role in the occurrence of vapochromism in non-fluorescent organic compounds. These reports suggest that

${ }^{a}$ Graduate School of Environmental and Information Sciences, Yokohama National University, 79-7 Tokiwadai, Hodogaya-ku, Yokohama 240-8501, Japan. E-mail: smatsu@ynu.ac.jp; Fax: +81-45-339-3345; Tel: +81-45-339-3366

${ }^{b}$ System Instruments Co., Ltd., 776-2 Komiya, Hachioji, Tokyo 192-0031, Japan

${ }^{c} X$-Ray Research Laboratory, Rigaku Corporation, 3-9-12 Matsubaracho, Akishima, Tokyo 196-8666, Japan

$\dagger$ Electronic supplementary information (ESI) available: experimental procedures and crystallographic data. CCDC 1479193. For ESI and crystallographic data in CIF or other electronic format see DOI: 10.1039/c6ra15966d the formation of solvates by exposure to solvent vapours contributes to the improvement of vapour sensing selectivity. Several organic vapoluminescent compounds containing an anthracene unit with bulky substituents were reported, exhibiting fluorescent properties that are tuned by inclusion of vapour molecules. ${ }^{7}$ However, this strategy is difficult to apply to the various well-known fluorophores due to the general occurrence of aggregation-caused quenching (ACQ) in crystalline organic dyes. ${ }^{8}$ In contrast, aggregation-induced emission (AIE) and crystallization-induced emission (CIE), which prevent the occurrence of ACQ, were also found to be an effective strategy for vapoluminescent materials, despite the fact that no inclusion of solvent molecules into the crystal structure upon exposure to solvent vapours was observed for the reported compounds exhibiting AIE and CIE. ${ }^{9}$ Additionally, many publications on the vapoluminescence were not included the detailed crystal structure data. Herein, we report the vapoluminescence of a purely organic 2,5-diamino-3,6-dicyanopyrazine dye, where the fluorophore-independent factors, i.e. bulky groups with high molecular flexibility and halogen substituents are essential for the occurrence of vapoluminescence. These two factors were found to contribute to the inhibition of fluorophore aggregation and favour the formation of solvate upon exposure to benzene vapour.

Compound 1 (2,5-bis[ $N, N$-di-(4-chlorophenyl)methylamino]3,6-dicyanopyrazine, Fig. 1) has five crystal forms with different colours. Among them, crystal structures of the three non-solvate forms (1Y, 1YO, and 1R) have been previously reported, ${ }^{10}$ although the relationship between crystal structures and measured optical properties were not found. These forms were crystallized from several solvent combinations, whereas the crystals of 10 were only obtained from benzene solution. ${ }^{10 b}$ The results of thermal analysis and powder X-ray diffraction (XRD) suggest that $\mathbf{1 0}$ can be regarded as a novel solvate form (Fig. S1 and S2†). Interestingly, powdered 10 could be immediately obtained by adding a small amount of benzene to powdered $1 \mathbf{Y}$ (Fig. S3 $\uparrow$ ). In addition, 10 was converted to $\mathbf{1 Y}$ by heating at $35^{\circ} \mathrm{C}$ for $c a .1 \mathrm{~h}$. It is also possible to obtain 10 by 
<smiles>N#Cc1nc(N(Cc2ccc(Cl)cc2)Cc2ccc(Cl)cc2)c(N(Cc2ccc(Cl)cc2)Cc2ccc(Cl)cc2)nc1[CH]COC(=O)OCc1ccccc1</smiles>

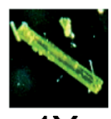

$1 Y$

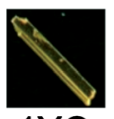

$1 Y 0$

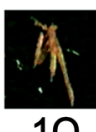

10

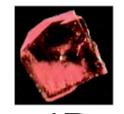

$1 \mathrm{R}$

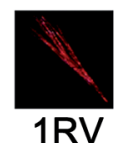

1RV
Fig. 1 Chemical structure of compound 1 and its five crystal forms.

treating $1 \mathrm{Y}$ with benzene vapour at room temperature (Fig. 2a and S4†). The absorption and emission maxima of $\mathbf{1 Y}$ in the visible region were observed at 484 and $558 \mathrm{~nm}$, respectively (Fig. 2b). The broad single peak was shifted to longer wavelengths upon exposure of $1 \mathrm{Y}\left(\lambda_{\max }=508 \mathrm{~nm}, F_{\max }=581 \mathrm{~nm}\right)$ to benzene vapour. We have previously reported that the crystal colour of 1Y was correlated with molecular deformation, particularly with changes of the amino group geometry, rather than with intermolecular interactions, such as exciton interaction. $^{10 a}$ The exciton interaction of $\mathbf{1 0}$ calculated by the expanded dipole model ${ }^{11}$ brought a negligible bathochromic shift of $c a .26 \mathrm{~cm}^{-1}$, since the distance between the fluorophores was large due to the bulky groups. In addition, both the absorption and emission spectra peaks and the high

(a)
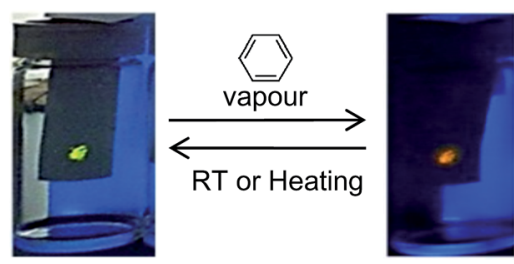

(b)

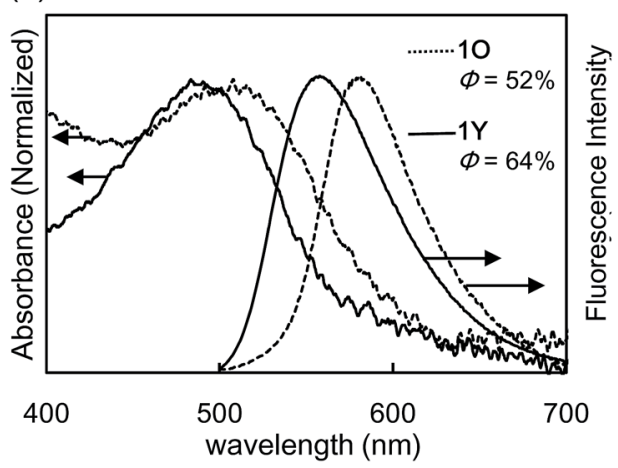

Fig. 2 (a) Colour change of $1 Y$ induced by exposure to benzene vapour. (b) Absorption and emission spectra of 10 and $1 Y$. fluorescence quantum yields of $\mathbf{1 O}$ and $\mathbf{1 Y}$ were similar to those of 1 in $\mathrm{CHCl}_{3}$ solution (Fig. S5†). These results strongly suggest that the electronic states of the monomer dominate the optical properties of 10 and $\mathbf{1 Y}$. The optical properties of the monomer in this crystalline dye are expected to correlate with the inhibition of molecular aggregation by the bulky groups.

Single-crystal XRD analysis revealed that the crystal structure of 10 exhibited a $1: 2$ ratio of compound 1 and benzene molecules (Table S1 $\dagger$ ). Conformational analysis indicated that the chromophore of $\mathbf{1 0}$, which contains a pyrazine ring, cyano groups, and amino groups, was relatively planar, similarly to $\mathbf{1 Y}$, whereas the amino group geometry of $\mathbf{1 0}$ was different from that of 1Y (Fig. 3a and b). The sum of the three angles around the amino nitrogen in 10 was $359.6^{\circ}$, thus the hybridisation of the nitrogen was regarded as $\mathrm{sp}^{2}$-like. In contrast, the hybridisation of the amino nitrogen of $\mathbf{1 Y}$ was considered as $\mathrm{sp}^{3}$ like. ${ }^{10 b}$ The dihedral angles between the amino group and the pyrazine ring in $1 \mathrm{O}$ and $1 \mathrm{Y}$ were $28.8^{\circ}$ and $43.8^{\circ}$, respectively. The amino groups of $\mathbf{1 0}$ are expected to be better electron donors for the chromophore than those of 1Y. The absorption spectra calculated by TD-DFT (B3LYP/6-31G*+LanL2DZ) based on each molecular conformation in the crystal structures of $\mathbf{1 0}$ and $1 \mathbf{Y}$ were consistent with the observed absorption spectra (Table S2 $\dagger$ ). The calculated absorption in the visible region for the two forms was assigned to the HOMO-LUMO transition. In 10 , the level of the HOMO, which has contributions from the $\pi$ electrons of the pyrazine ring and the lone pairs of amino groups, was destabilized compared to that of $\mathbf{1 Y}$, whereas the LUMOs of both forms had essentially the same energy (Fig. 3c (a)

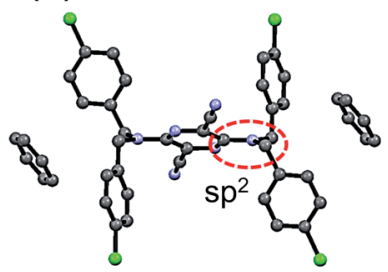

(c)

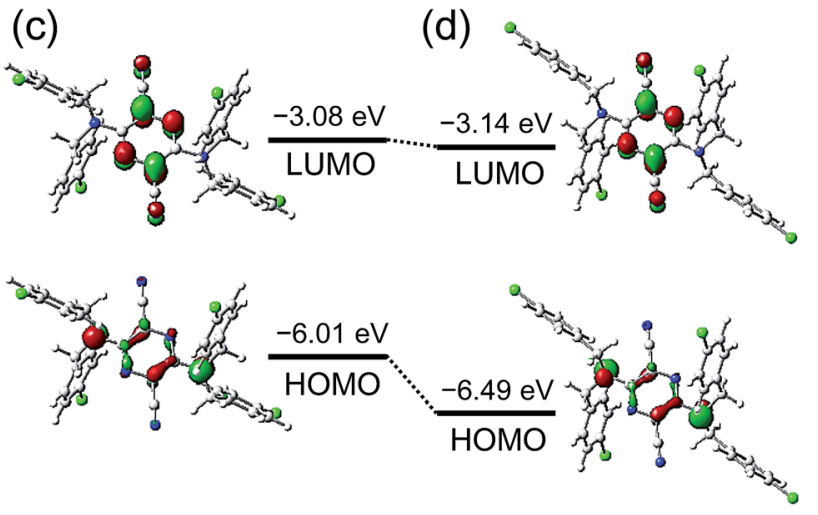

Fig. 3 Conformational difference between (a) 10 and (b) $1 Y$ in the observed crystal structures. The red dashed line represents the amino group. (c) Highest occupied molecular orbital (HOMO) and lowest unoccupied molecular orbital (LUMO) of 10 and (d) 1Y calculated by TD-DFT. 
and d). Particularly, the HOMOs of the two forms had differing contributions of the amino groups, revealing that the different colour of $\mathbf{1 O}$ and $\mathbf{1 Y}$ originated from their deformation.

To understand the mechanism of benzene inclusion, we compared the crystal structures of $\mathbf{1 O}$ and $\mathbf{1 Y}$. In the former, the dye molecules formed 1-D chromophore stacking columns, with benzene molecules positioned between these columns, more specifically, between benzyl groups (Fig. 4a). The spaces occupied benzene molecules in the crystal structure of $\mathbf{1 0}$ were not seen for $\mathbf{1 Y}$, although the dye molecules in $\mathbf{1 0}$ and $\mathbf{1 Y}$ were found to form the same 1-D chromophore stacking columns. ${ }^{10 b}$ The presence or absence of the spaces in the crystal structure resulted from the subtle conformational difference between the two forms in the position of the benzyl group (Fig. 4b). In other words, this difference generated the spaces between the 1-D chromophore stacking columns. The benzyl group in $\mathbf{1 Y}$ can move to the position corresponding to $\mathbf{1 0}$ upon exposure to benzene vapour (Fig. 4c), which contributes to the generation of suitable spaces for the inclusion of benzene molecules in the crystal structure of $\mathbf{1 0}$ (Fig. 4d). One of the possible driving forces of the conformational change was the difference in the thermodynamical stability of these two conformations. The calculation of conformational energy in the gas phase using $\mathrm{B} 3 \mathrm{LYP} / 6-31 \mathrm{G}^{*}$ resulted that the conformation of $\mathbf{1 0}$ was more stable than that of $\mathbf{1 Y}$. The energy difference was $4.9 \mathrm{kcal} \mathrm{mol}^{-1}$. In contrast, the lattice energy calculated by the atom-atom Coulomb-London-Pauli model ${ }^{\mathbf{1 2}}$ revealed that the crystal structure of $\mathbf{1 O}$ was less stable than that of 1Y. The energy difference was $31.3 \mathrm{kcal} \mathrm{mol}^{-1}$. These results indicated that the more stable crystal form was $\mathbf{1 Y}$. However, when the intermolecular interactions of the crystal packing of $\mathbf{1 Y}$ become weaker

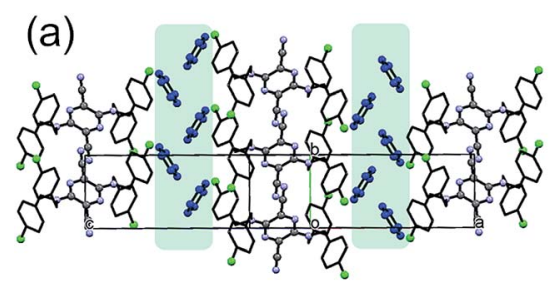

(b)
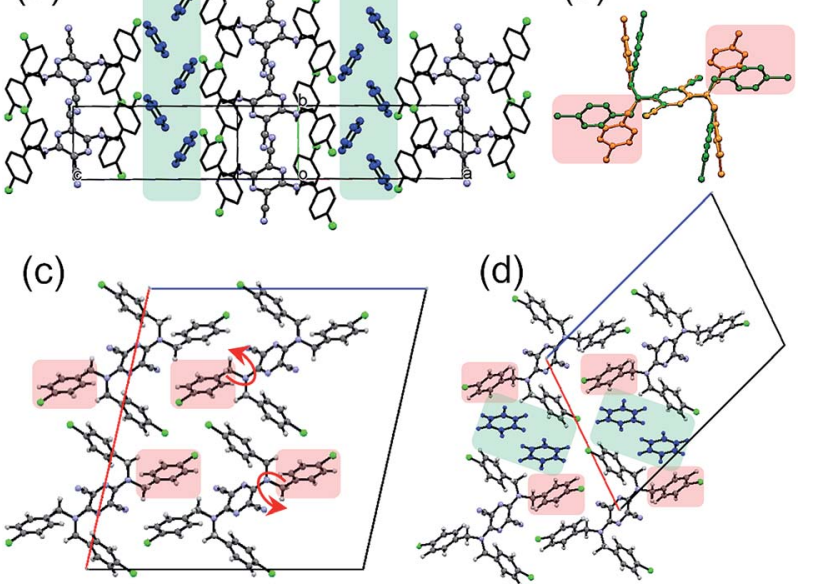

(d)

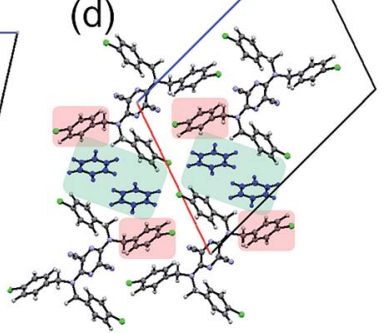

Fig. 4 (a) Crystal structure of 10. The benzene molecules (shown in blue) are packed between the 1-D chromophore stacking columns (shown in green squares). (b) Conformational difference between the benzyl groups of 10 (shown in orange) and $1 Y$ (shown in green). The red squares represent a significantly different shape of the benzyl group in the two motifs. (c) Crystal structures of $1 Y$ and (d) 10 viewed down the $b$-axis. The red squares in (c) and (d) indicate a significantly different shape of the benzyl group compared to (b). The benzyl groups of $1 Y$ presumably moved in the direction of the red arrows in (c) upon exposure to benzene vapour. by external stimuli such as exposure to solvent vapour, the conformation of $\mathbf{1 Y}$ can convert to that of $\mathbf{1 0}$ by the conformational energy difference. Furthermore, weak interactions played an important role in the stabilization of the conformational deformation by exposure to benzene vapour. In the intermolecular interactions between the 1-D chromophore stacking columns (Fig. S6 $\dagger$ ), there found the intermolecular C$\mathrm{H} \cdots \pi / \mathrm{C}$ interaction and weak interactions such as $\mathrm{C}-\mathrm{H} \cdots \mathrm{Cl}$ and $\mathrm{C}-\mathrm{Cl} \cdots \pi / \mathrm{C}$ interactions based on the short contacts in 10, whereas such intermolecular interactions were not observed in $1 Y$ (Table S3†). The interaction energy of a molecular pair between the 1-D columns of $10\left(-10.0 \mathrm{kcal} \mathrm{mol}^{-1}\right)$ was lower than that of $\mathbf{1 Y}\left(-7.3 \mathrm{kcal} \mathrm{mol}^{-1}\right)$, although the distance between the molecular pair of 10 (11.670 ̊) was almost the same as that of $\mathbf{1 Y}(11.684 \AA)$. In addition, the proportion of coulombic term in the interaction energy in $10(10.8 \%)$ was larger than that of $\mathbf{1 Y}(6.9 \%)$. These results suggested that the electrostatic attraction by weak interactions contributed to the stabilization of the conformational deformation by benzene vapour in the crystalline state. Particularly, weak interactions involving the terminal halogen substituents of the benzyl groups can play a significant role in the inclusion of benzene molecules via the conformational deformation. This hypothesis was experimentally supported by examining an analogous crystalline dye $\mathbf{2 Y}$, which is the yellow crystal form of a compound $\mathbf{1}$ derivative with methyl groups in place of $\mathrm{Cl}$ atoms. 2Y did not exhibit vapochromism upon exposure to benzene vapour (Fig. S7 $\dagger$ ), despite its crystal structure being isomorphous with that of $\mathbf{1 Y} .^{\mathbf{1 0 b}}$ Moreover, no vapochromism upon exposure to benzene vapour was also observed for $\mathbf{3 Y}$, which is the yellow crystal form of a compound $\mathbf{1}$ derivative with $\mathrm{Br}$ atoms in place of $\mathrm{Cl}$ atoms (Fig. S7 $\dagger$ ). The molecular conformations of $\mathbf{3 Y}$ and $\mathbf{1 Y}$ were the same, whereas their crystal structures exhibited a subtle difference in benzyl group packing. ${ }^{10 b}$ These results suggest that both the weak interactions involving halogens and the packing of benzyl groups have a great impact on the formation of spaces for benzene molecules in the crystal structures. As a result, the ability to exhibit vapochromism is mainly influenced by the terminal substituents of the benzyl groups as well as by conformational flexibility of the pyrazine dye.

Exposure of 1Y to vapours of solvents other than benzene did not induce a reversible change of colour (Fig. S8a†). Pyridine, cyclohexane, and chloroform vapours induced an irreversible colour change of $\mathbf{1 Y}$ from yellow to orange (Fig. S8b†). The crystals of 1Y presumably dissolved in these vapours due to the high solubility of $\mathbf{1 Y}$ and the high vapour pressure of these solvents. This observation suggested that the spaces for solvent molecules of the crystal structure could not be varied by solvent size. In addition, the strength of the interactions between the pyrazine dye and solvent molecules influenced the formation of solvates upon exposure to solvent vapours.

\section{Conclusions}

In summary, exposure to benzene vapour led to a reversible change in the optical properties of the yellow form of the 
pyrazine dye. The change of crystal colour resulted from the deformation of the molecular conformation. The flexibility of the benzyl groups and the interactions involving their terminal substituents played an important role in the formation of the benzene solvate. These results indicated that the flexible bulky benzyl groups with halogen substituents can contribute to the development of novel organic vapoluminescent materials without metal atoms.

\section{Acknowledgements}

This research was partially supported by the Sasakawa Scientific Research Grant from The Japan Science Society.

\section{Notes and references}

1 (a) J. R. Askim, M. Mahmoudi and K. S. Suslick, Chem. Soc. Rev., 2013, 42, 8649; (b) Z. Chi, X. Zhang, B. Xu, X. Zhou, C. Ma, Y. Zhang, S. Liu and J. Xu, Chem. Soc. Rev., 2012, 41, 3878; (c) M. Irie, Chem. Rev., 2000, 100, 1685; (d) A. Fihey, A. Perrier, W. R. Browne and D. Jacquemin, Chem. Soc. Rev., 2015, 44, 3719; (e) C. Reus and T. Baumgartner, Dalton Trans., 2016, 45, 1850.

2 (a) M. Hakim, Y. Y. Broza, O. Barash, N. Peled, M. Philips, A. Amann and H. Haick, Chem. Rev., 2012, 112, 5949; (b) M. Phillips, K. Gleeson, J. M. B. Hughes, J. Greenberg, R. N. Cataneo, L. Baker and W. P. McVay, Lancet, 1999, 353, 1930; (c) W. Miekisch, J. K. Schubert and G. F. E. Noeldge-Schomburg, Clin. Chim. Acta, 2004, 347, 25. 3 (a) X. Zhang, B. Li, Z. H. Chen and Z. N. Chen, J. Mater. Chem., 2012, 22, 11427; (b) O. S. Wenger, Chem. Rev., 2013, 113, 3686.

4 H. Xia, D. Liu, K. Song and Q. Miao, Chem. Sci., 2011, 2, 2402. 5 E. Takahashi, H. Takaya and T. Naota, Chem.-Eur. J., 2010, 16, 4793.

6 (a) M. Kaftory, H. Taycher and M. Botoshansky, J. Chem. Soc., Perkin Trans. 2, 1998, 407; (b) K. Fujii, A. Sakon, A. Sekine and
H. Uekusa, Cryst. Growth Des., 2011, 11, 4305; (c) J. L. Scott, A. Almesaker, Y. Sumi and K. Tanaka, Cryst. Growth Des., 2007, 7, 1049.

7 (a) H. Naito, Y. Morisaki and Y. Chujo, Angew. Chem., Int. Ed., 2015, 54, 5084; (b) P. Rajamali, P. Gandeepan, M. J. Huang and C. H. Cheng, J. Mater. Chem. C, 2015, 3, 3329; (c) T. H. Brehmer, P. P. Korkas and E. Weber, Sens. Actuators, 1997, B44, 595.

8 (a) J. R. Lakowicz, Principles of Fluorescence Spectroscopy, Springer, 3rd edn, 2006; (b) Y. Hong, J. W. Y. Lam and B. Z. Tang, Chem. Commun., 2009, 4332.

9 (a) M. Tanioka, S. Kamino, A. Muranaka, Y. Ooyama, H. Ota, Y. Shirasaki, J. Horigome, M. Ueda, M. Uchiyama, D. Sawada and S. Enomoto, J. Am. Chem. Soc., 2015, 137, 6436; (b) X. Luo, W. Zhao, J. Shi, C. Li, Z. Liu, Z. Bo, Y. Q. Dong and Z. Tang, J. Phys. Chem. C, 2012, 116, 21967; (c) Z. He, L. Zhang, J. Mei, T. Zhang, J. W. Y. Lam, Z. Shuai, Y. Q. Dong and B. Z. Tang, Chem. Mater., 2015, 27, 6601; (d) B. K. An, S. K. Kwon, S. D. Jung and S. Y. Park, J. Am. Chem. Soc., 2002, 124, 14410; (e) B. K. An, S. K. Kwon and S. Y. Park, Bull. Korean Chem. Soc., 2005, 26, 1555; (f) S. J. Yoon, J. W. Chung, J. Gierschner, K. S. Kim, M. G. Choi, D. Kim and S. Y. Park, J. Am. Chem. Soc., 2010, 132, 13675; (g) M. S. Yuan, D. E. Wang, P. Xue, W. Wang, J. C. Wang, Q. Tu, Z. Liu, Y. Zhang and J. Wang, Chem. Mater., 2014, 26, 2467; (h) P. S. Hariharan, D. Moon and S. P. Anthony, J. Mater. Chem. C, 2015, 3, 8381.

10 (a) S. Matsumoto, Y. Uchida and M. Yanagita, Chem. Lett., 2006, 35, 654; (b) Y. Akune, H. Gontani, R. Hirosawa, A. Koseki and S. Matsumoto, CrystEngComm, 2015, 17, 5789. CSD refcode: 1R (KELFOX), 1Y (KELFOX01), 1YO (KELFOX02), 2 Y (HUMFIG), 3 Y (KELGAK).

11 T. Kobayashi, J-Aggregates, World Scientific Publishing, 1996, pp. 1-40.

12 A. Gavezzotti, New J. Chem., 2011, 35, 1360. 